\title{
Frecuencia de consultas cardiológicas y paros cardíacos extrahospitalarios durante la pandemia COVID-19 en Uruguay
}

\author{
Dres. Víctor Dayan,2, Abayubá Perna³, Natalia Piñeiro33, Álvaro Niggemeyer ${ }^{1,2}$
}

\section{Resumen}

Introducción: la pandemia COVID-19 ha determinado la aplicación de medidas sanitarias de emergencia, tendientes a evitar la progresión a nivel nacional. Reportes internacionales han sugerido que dichas medidas determinaron secundariamente una desatención en otras patologías, principalmente cardiovasculares, y eventual aumento de paros cardíacos extrahospitalarios (PCEH).

Objetivo: determinar la frecuencia de consultas en emergencia por dolor precordial a nivel nacional y de PCEH asistidos por los principales servicios de emergencia prehospitalaria (SEP) de Montevideo, durante la pandemia.

Método: se solicitó información del número de consultas por dolor precordial y de consultas totales, a las instituciones médicas de Montevideo y del interior del país, realizadas en el período comprendido entre el 13 de marzo y el 30 de abril de los años 2018, 2019 y 2020. Se solicitó a los SEP información sobre la frecuencia de PCEH en Montevideo durante los mismos períodos. Los datos se expresan como frecuencia absoluta y tasa de incidencia (por 100.000 afiliados) con su intervalo de confianza estimado mediante Fisher.

Resultados: el número de consultas por dolor precordial fue estable durante el período 2018-2019. Durante 2020, dichas consultas disminuyeron, representando entre 11,3\% y 21,7\% del total de consultas. Se evidenció un aumento no significativo en la tasa de PCEH en el 2020 (9,05, IC95\%: 7,15-11,30) comparado con el 2019 (7,94, IC95\%: 6,19-10,04) y 2018 (7,43, IC95\%: 5,75-9,45).

Conclusiones: los datos crudos presentados muestran que desde el 13 de marzo hasta el 30 de abril de 2020 hubo una disminución en las consultas en emergencia por dolor precordial, aumentando de forma no significativa el número de PCEH durante el mes de abril, respecto al mismo período de los dos años anteriores.

Palabras clave:

COVID-19

DOLOR PRECORDIAL

PARO CARDÍACO EXTRAHOSPITALARIO

\section{Frequency of cardiological consultations and out-of-hospital cardiac arrests during the COVID-19 pandemic}

\section{Summary}

Introduction: the COVID-19 pandemic has determined the application of emergency health measures aimed at preventing progression at national level. International reports have suggested that these measures lead to a lack of care in other pathologies, mainly cardiovascular, and eventually increase out-of-hospital cardiac arrests.

Objective: to determine the frequency of emergency consultation for chest pain and out-of-hospital cardiac arrests, assisted by the main pre-hospital emergency services of Montevideo, during the COVID-19 pandemic.

Methods: information was requested to the medical institutions of Montevideo and all over the country, on the number of consultations for chest pain and total consultations, in the period March 13- April 30 of 2018, 2019 and 2020. The frequency of out-of-hospital cardiac arrests in Montevideo was requested to the pre-hospital emergency services during the same period. The data is expressed as absolute frequency and incidence rates (x 100,000) with its 95\% CI calculated by Fisher.

1. Centro Cardiovascular Universitario. Universidad de la República. Montevideo, Uruguay.

2. Sociedad Uruguaya de Cardiología. Montevideo, Uruguay.

3. Fondo Nacional de Recursos. Montevideo, Uruguay.

Correspondencia: Dr. Víctor Dayan. Correo electrónico: victor_dayan@hotmail.com

Los autores declaran no tener conflictos de intereses.

El presente estudio no tiene fuentes de financiamiento.

Recibido Jun 8, 2020; aceptado Jun 18, 2020. 
Results: the number of consultations for precordial pain was stable during the 2018-2019 period. During 2020, these consultations decreased and represented between $11.3 \%$ and $21.7 \%$ of the total number of consultations. Out-of-hospital cardiac arrests showed a non significant increase in its incidence rate in 2020 (9.05, 95\%IC: 7.15-11.30) compared with 2019 (7.94, 95\%IC: 6.19-10.04) and 2018 (7.43, 95\%IC: 5.75-9.45).

Conclusions: the raw data presented shows that from March 13 to April 30 of 2020, there was a decrease in emergency visits for precordial pain and a non-significant increase in the incidence rate of out-of-hospital cardiac arrests.

Key words: $\quad$ COVID-19

CHEST PAIN

OUT OF HOSPITAL CARDIAC ARREST

\section{Frequência de consultas cardiológicas e paradas cardíacas fora do hospital durante a pandemia de COVID-19}

\section{Resumo}

Introdução: a pandemia do COVID-19 determinou a aplicação de medidas emergenciais de saúde destinadas a impedir a progressão em nível nacional. Relatórios internacionais sugerem que essas medidas levam à falta de atendimento em outras patologias, principalmente cardiovasculares, e eventualmente aumentam as paradas cardíacas fora do hospital. Objetivo: determinar a frequência da consulta de emergência para dor no peito e paradas cardíacas fora do hospital, assistidas pelos principais serviços de emergência pré-hospitalar de Montevidéu, durante a pandemia do COVID-19.

Métodos: foram solicitadas informações às instituições médicas de Montevidéu e de todo o país sobre o número de consultas para dor no peito e total de consultas, no período de 13 de março a 30 de abril de 2018, 2019 e 2020 . A frequência de -as paradas cardíacas no hospital foram solicitadas aos serviços de emergência pré-hospitalares durante o mesmo período. Os dados são expressos como frequência absoluta e taxa de incidência (x 100.000) com seu intervalo de confiança estimado por Fisher.

Resultados: o número de consultas para dor precordial permaneceu estável no período 2018-2019. Durante 2020, essas consultas representaram entre $11,3 \%$ e $21,7 \%$ do número total de consultas. Um aumento não significativo da taxa de paradas cardíacas fora do hospital foi evidente em 2020 (9,05, IC 95\%: 7,15-11,30) em comparação com 2019 (7,94, IC 95\%: 6,19-10,04) e 2018 (7,43, 95\%IC: 5,75-9,45), o que não é significativo.

Conclusóes: os dados brutos apresentados mostram que de 13 de março a 30 de abril de 2020 houve uma diminuição nas visitas de emergência por dor precordial, não aumentando significativamente o número de paradas cardíacas fora do hospital em comparação aos dois anos anteriores.

Palavras chave: COVID-19

DOR NO PEITO

PARAGEM CARDÍACA EXTRA HOSPITALAR

\section{Introducción}

En diciembre de 2019 se reporta el primer brote del síndrome respiratorio agudo ocasionado por el coronavirus-2 (SARS-CoV-2), en China ${ }^{(1)}$. Posteriormente, el 11 de marzo de 2020 la Organización Mundial de la Salud (OMS) lo declara pandemia glo$\mathrm{bal}^{(2)}$. Desde su emergencia y hasta la fecha se han reportado mundialmente más de 8 millones de casos y 450.000 muertes.

El 13 de marzo de 2020, se registra el primer grupo de casos positivos para SARS-CoV-2 en Uruguay, y el gobierno de forma inmediata implementa la emergencia sanitaria. Esta consistió, entre otras, en medidas progresivas destinadas al distanciamiento físico. Las políticas implementadas lograron limitar de forma significativa la dispersión del virus, logrando las cifras de contagio más bajas de toda América del Sur.

Los contagios y la enfermedad resultante lograron ser controlados; sin embargo, los efectos colaterales e indirectos de la emergencia sanitaria son as-
Principales aportes

- A partir de marzo de 2020 y a raíz de la pandemia por coronavirus, se vive un escenario de situación sanitaria inédito.

- Distintos factores podrían determinar desatención en ciertas patologías, como las cardiovasculares.

- Diversos autores plantean una disminución en el número de consultas por ángor durante la pandemia y su eventual relación con el aumento de los paros cardíacos extrahospitalarios y la disminución de infartos de miocardio tratados.

- En el presente trabajo se detectó una disminución importante del número de consultas por ángor durante la pandemia, en todo el país, así como un ligero aumento no significativo en la incidencia de paros cardíacos extrahospitalarios en Montevideo. 
Tabla 1. Número de consultas por dolor precordial por centro médico durante el mes de abril de los años 2018, 2019 y 2020. Los porcentajes representan la proporción para cada año sobre el total de consultas por dolor precordial de cada centro.

\begin{tabular}{|c|c|c|c|c|}
\hline Centro médico & $2018(\%)$ & $2019(\%)$ & $2020(\%)$ & Total \\
\hline A & $341(39,8)$ & $329(38,4)$ & $186(21,7)$ & $856(100)$ \\
\hline B & $185(37,6)$ & $227(46,1)$ & $80(16,3)$ & $492(100)$ \\
\hline C & $324(40,2)$ & $307(38,1)$ & $175(21,7)$ & $806(100)$ \\
\hline $\mathrm{D}$ & $46(46,9)$ & $41(41,8)$ & $11(11,2)$ & $98(100)$ \\
\hline $\mathrm{E}$ & $24(51,1)$ & $17(36,2)$ & $6(12,8)$ & $47(100)$ \\
\hline $\mathrm{F}$ & $158(41,8)$ & $164(43,4)$ & $56(14,8)$ & $378(100)$ \\
\hline
\end{tabular}

pectos de preocupación a nivel mundial(3). Dentro de los efectos de mayor preocupación están aquellos vinculados a la desatención de las patologías cardiovasculares $^{(4)}$. Varios autores han sugerido que la disminución en la incidencia de infartos diagnosticados y tratados durante la pandemia, puede ser causa de una mayor tasa de paros cardíacos extrahospitalarios $(\mathrm{PCEH})^{(3,5)}$.

Apremiados por la emergencia de la situación, nos propusimos realizar un diagnóstico rápido de situación relacionado a la frecuencia en la atención de los pacientes con dolor precordial y PCEH.

\section{Método}

Se trata de un estudio descriptivo de tipo ecológico, realizado mediante el aporte de datos de las principales instituciones sanitarias de todo el país y emergencias móviles de Montevideo que accedieron a proporcionar los datos solicitados.

Desde la Sociedad Uruguaya de Cardiología contactamos a 50 centros médicos y servicios de emergencia prehospitalaria (SEP), solicitando información referente al número de consultas presenciales por dolor en el pecho desde el 13 de marzo hasta el 30 de abril de 2020. Concomitantemente, solicitamos la misma información para el mismo período de los años 2018 y 2019. Se solicitó a los SEP información respecto al número de $\mathrm{PCEH}$ asistidos durante el mes de abril en cada uno de los años. Las SEP que participaron del estudio fueron SEMM, UCM, SUAT y Emergencia UNO. De las instituciones médicas que decidieron participar, dado que no todas consintieron en hacer público su nombre, se mantienen en forma anónima (seis en total).

Se solicitó el número de afiliados de cada SEP para poder calcular tasas de incidencia de PCEH. Se calculó una tasa de paro cardiorrespiratorio por 100.000 socios para el mes abril por cada SEP y globales por año (2018, 2019 y 2020). Para las tasas globales por año se calcularon intervalos de confian- za (IC) a 95\% usando la fórmula de Fisher. Dichos datos se expresan como tasa de incidencia por 100.000 afiliados. Para definir la significancia en cuanto a la diferencia de tasas entre los años considerados, se valoró el valor puntual de la tasa durante el mes de abril de 2020 y se identificó si la misma estaba comprendida dentro del $95 \%$ IC de los años anteriores. Se consideró significativo una $\mathrm{p}<0,05$.

Dado que se trata de un estudio poblacional con datos demográficos, sin identificación de los pacientes, no requiere aprobación de Comité de Ética.

\section{Resultados}

El número de consultas por dolor precordial en las emergencias de las instituciones participantes fue bastante estable durante el período 2018-2019, y se evidencia un descenso importante en 2020 (tabla 1, figura 1). En el período de 2020, las consultas por dolor precordial constituyeron entre $11,2 \%$ y $21,7 \%$ del total de dichas consultas de los años 2018 a 2020.

Respecto al número de PCEH asistidos, la frecuencia ha sido variable a lo largo de los años para los cuatro SEP. En su globalidad, la frecuencia absoluta evidencia un ligero aumento respecto a años anteriores (figura 2).

El número de afiliados reportados por los SEP para 2018 fue de 888.225; en 2019 de 881.283, y en 2020 de 861.779 individuos.

La tasa de incidencia de PCEH durante 2020 fue de 9,05 (IC95\%: 7,15-11,30); para 2019 de 7,94 (IC95\%: 6,19-10,04), y para 2018 de 7,43 (IC95\%: $5,75-9,45)$. Dado que el valor puntual de 2020 está incluido dentro de los IC de 2019 y 2018 , concluimos que no hubo diferencias significativas durante 2020 (tabla 2, figura 3).

\section{Discusión}

Los datos crudos muestran una clara reducción en el número de consultas en emergencia por dolor 


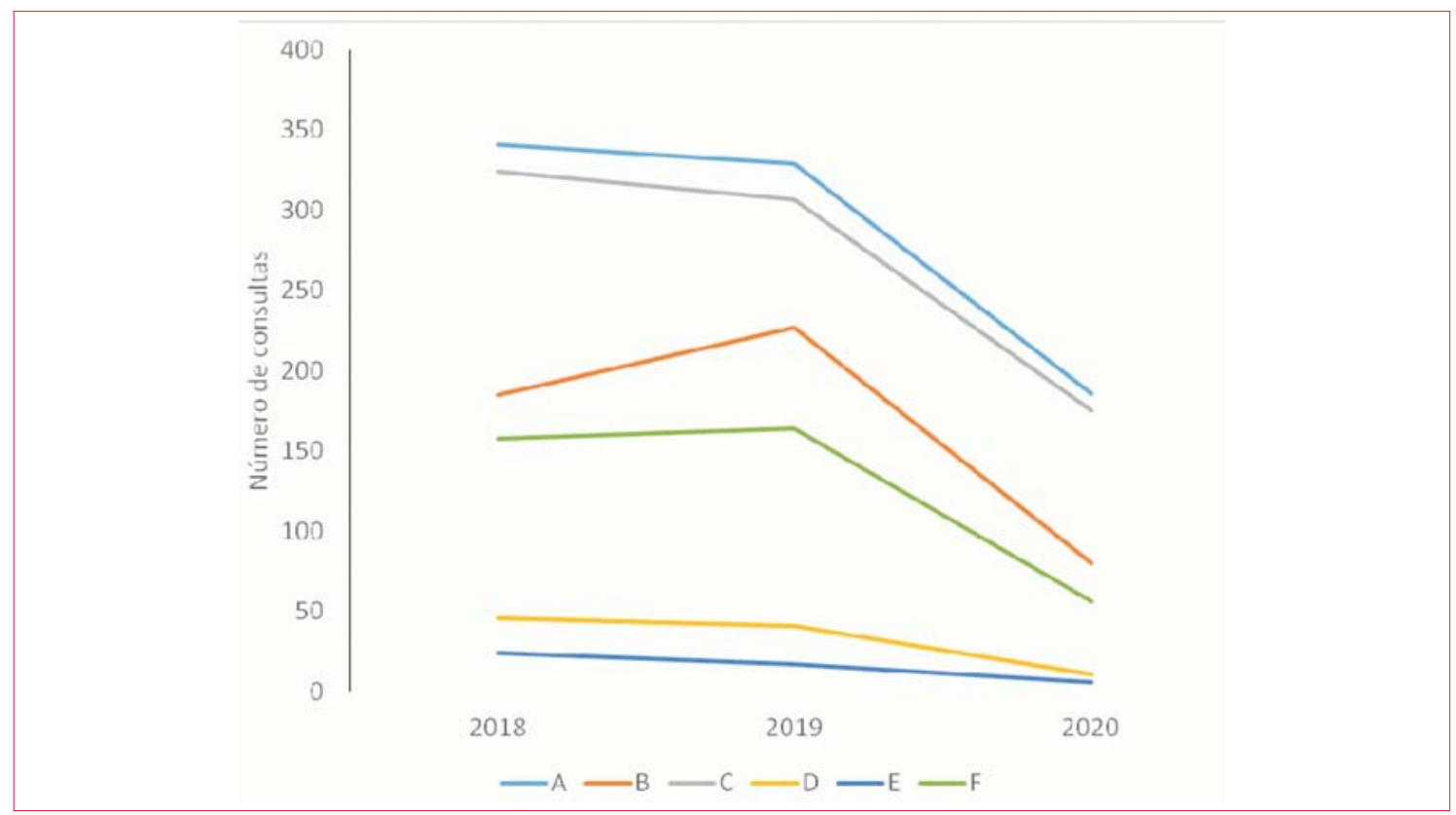

Figura 1. Frecuencia de consultas por dolor precordial durante el mes de abril de 2018, 2019 y 2020 en los distintos centros médicos. Cada color y letra representa uno de los centros médicos participantes del estudio.

Tabla 2. Tasa de incidencia global de PCEH durante el mes de abril, según reporte de SEP.

\begin{tabular}{lccc}
\hline Año & Tasa por 100.000 & IC95\% & Población expuesta \\
\hline 2018 & 7,43 & $5,75-9,45$ & 888.225 \\
2019 & 7,94 & $6,19-10,04$ & 881.283 \\
2020 & 9,05 & $7,15-11,30$ & 861.779 \\
\hline
\end{tabular}

PCEH: paro cardíaco extrahospitalario; SEP: servicio de emergencia prehospitalaria.

precordial. Aunque en nuestro medio existía esa "sensación", esta es la primera vez que se reporta objetivamente. Esta reducción plantea dudas respecto a la ocurrencia de eventos agudos coronarios no diagnosticados, $\mathrm{y}$, por ende, no tratados, o la hipótesis alternativa que relaciona el estilo de vida impuesto por la emergencia sanitaria, a un menor riesgo de eventos cardiovasculares.

Existen dos grandes estudios poblacionales publicados acerca de la incidencia de PCEH durante la pandemia COVID-19(1,2). Al día de hoy, Francia reporta 153.000 casos positivos para SARS-CoV-2, e Italia 235.000. En Uruguay, al momento de este reporte, el número total de casos diagnosticados es de 832, con menos de 100 casos activos actualmente. Considerando este bajo número, podemos asumir que el comportamiento del PCEH durante el período estudiado en nuestro país obedece fundamentalmente a las medidas de emergencia sanitaria y no al efecto directo de la enfermedad COVID-19.
Existe una gran preocupación acerca de la desatención de otras patologías diferentes a COVID-19 durante la pandemia. Se ha reportado un menor número de tratamientos de infarto agudo de miocardio, lo que puede deberse a que los propios pacientes no consultan, y que determinaría una mayor mortalidad cardiovascular ${ }^{(4)}$. Aunque dicho dato no ha sido presentado hasta la fecha en ninguna publicación, varios autores han trasladado este probable impacto en la cuantificación de PCEH. En París, se ha reportado una duplicación en la tasa de $\mathrm{PCEH}$ durante la pandemia y en Italia un aumento de $58 \%$. Entre $77 \%$ y $33 \%$ de los individuos que explican el aumento de la incidencia de los PCEH reportados en Italia y Francia, respectivamente, tenían infección por el nuevo coronavirus ${ }^{(3,5)}$.

Los reportes provenientes de los SEP más importantes de Montevideo incluyen a más de 800.000 individuos, lo que representa una gran proporción de la población capitalina (62\%). Los datos presen- 


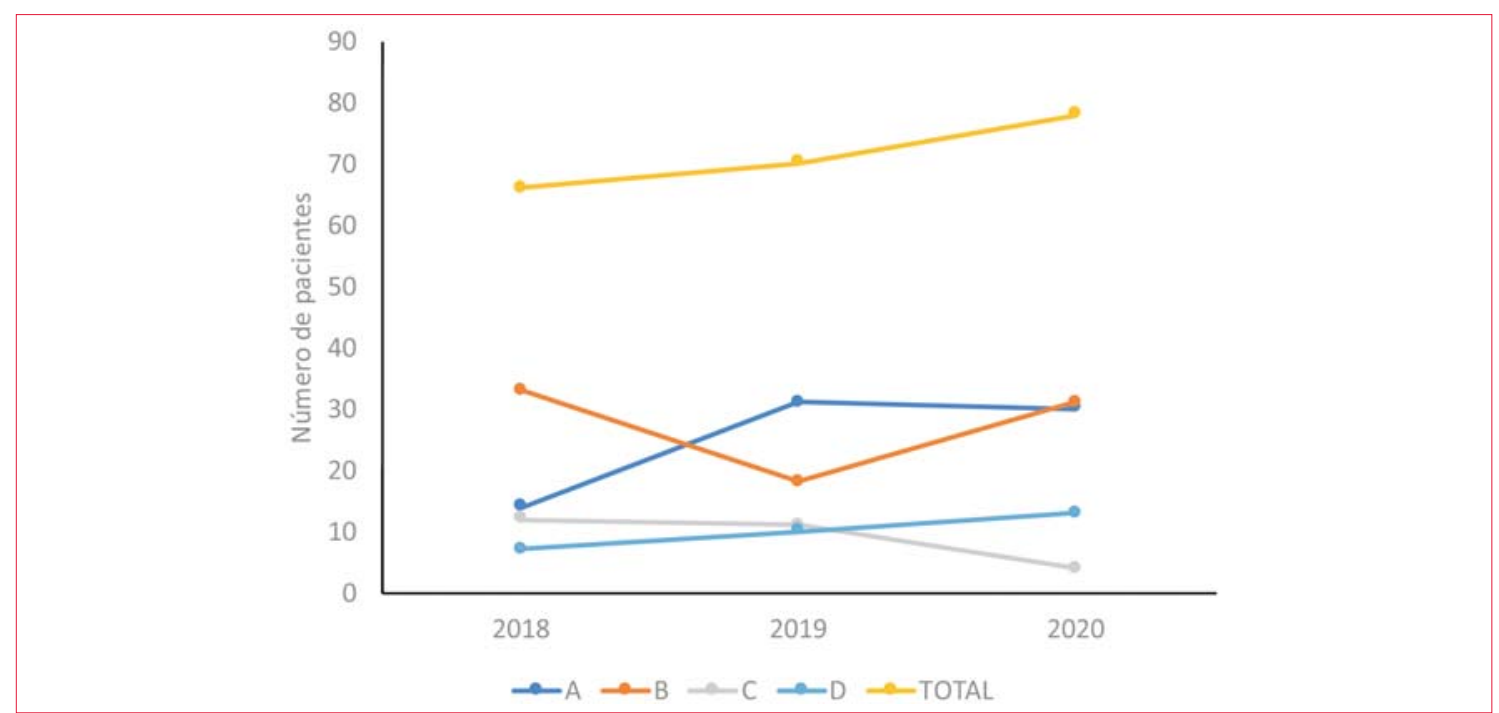

Figura 2. Frecuencia de paros cardíacos extrahospitalarios durante el período de estudio de 2018, 2019 y 2020 asistidos por los distintos servicios de emergencia prehospitalaria.

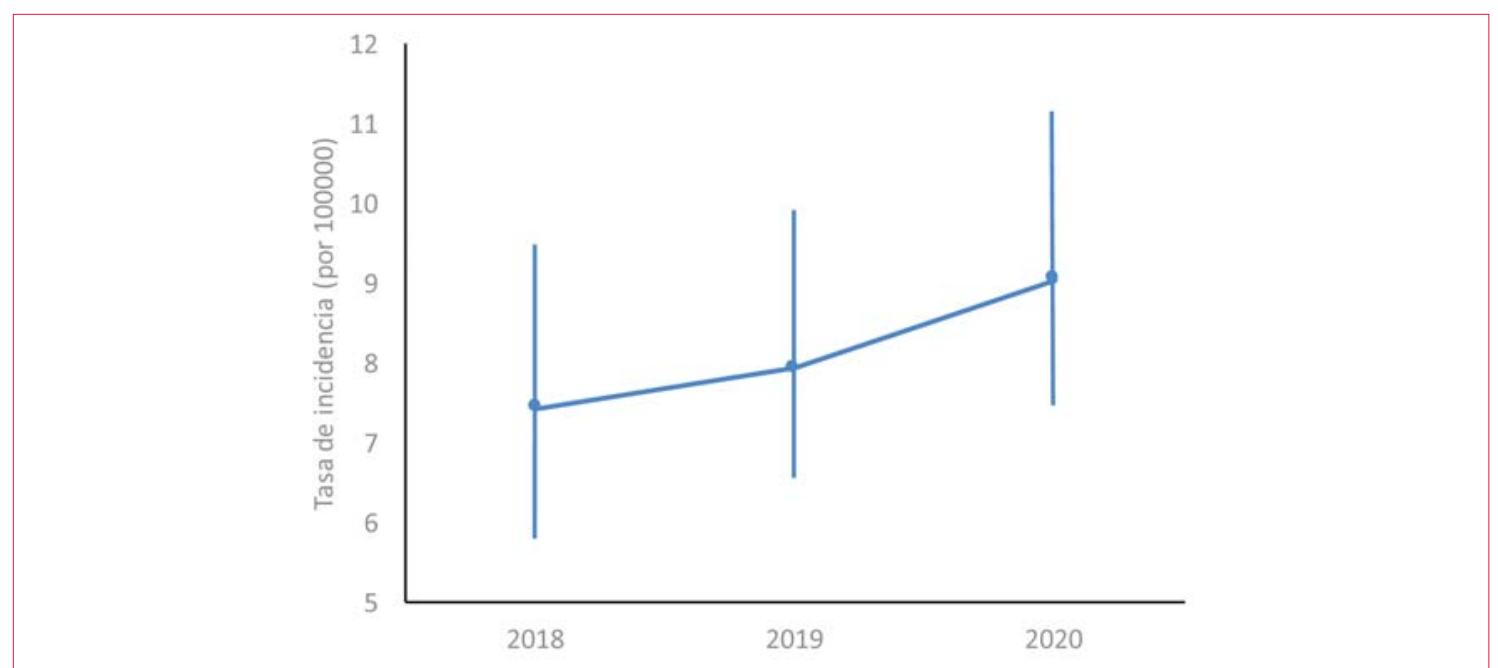

Figura 3. Tasa de paro cardiorrespiratorio por 100.000 afiliados según año (mes de abril). Las barras representan el $95 \%$ de IC.

tados muestran que el comportamiento fue heterogéneo y no se evidenció un pico en frecuencia de PCEH, como sí fue reportado en otros países ${ }^{(3,5)}$. Con estos datos no encontramos un aumento significativo en la tasa de incidencia de PCEH. Según un reporte del Ministerio de Salud Pública divulgado en los medios de prensa(6), la mortalidad global disminuyó ligeramente durante este período. Sin embargo, para poder completar el análisis del impacto de la emergencia sanitaria en la patología cardiovascular, es necesario integrar el estudio de la tasa de infartos, PCEH y mortalidad cardiovascular.

\section{Limitaciones}

Los datos aquí presentados tienen grandes limitaciones, dentro de las que destacan la imposibilidad de determinar la tasa real de incidencia de PCEH. Esto se debe a que el número de afiliados de los SEP puede incluir individuos que poseen más de un SEP (lo cual sobrestima el denominador), así como también pueden contabilizarse de forma distinta los afiliados dentro de cada SEP debido a convenios existentes en el momento. Sin embargo, el objetivo real del presente reporte no es el dato aislado de incidencia, sino su variación a lo largo del tiempo. A pesar de incluir un porcentaje significativo de la población de Montevideo, no tenemos los datos totales. El no tener información respecto al "estado COVID-19" de los individuos fallecidos, nos inhabilita a hacer conclusiones sobre el efecto de este en los PCEH. Cada emergencia móvil codifica los llamados totales de forma diferente, razón por lo cual decidimos no incluir dicho dato en el análisis. Respecto al número 
de consultas en puerta de emergencia, las autoridades sanitarias recomendaron evitarlas (sustituyéndolas por consultas en SEP) para prevenir aglomeraciones, lo que puede contribuir a explicar las disminuciones de estas por dolor precordial.

A pesar de las limitaciones presentadas, considerando y asumiendo una población de afiliados fija con un denominador sobrestimado de forma constante a lo largo de los períodos considerados, el comportamiento longitudinal en su globalidad nos permite sugerir que las medidas de emergencia sanitaria implementadas no han impactado de forma significativa en el número de PCEH.

\section{Conclusiones}

Los datos crudos presentados muestran que desde el 13 de marzo hasta el 30 de abril de 2020 hubo una disminución en las consultas en emergencia por dolor precordial, aumentando de forma no significativa el número de $\mathrm{PCEH}$ en Montevideo durante el mes de abril, respecto al mismo período de los dos años anteriores.

Contribución de autores

Víctor Dayan, https://orcid.org/0000-0002-5470-0585

Idea original, concepción y diseño del trabajo; adquisi-

ción, análisis e interpretación de los datos, revisión y edición del manuscrito final. Comunicación con editores.

Aprobación de la versión final a ser publicada.

Abayubá Perna, https://orcid.org/0000-0001-7062-1310 Adquisición, análisis e interpretación de los datos, revisión y edición del manuscrito final. Aprobación final de la versión a ser publicada.

Natalia Piñeiro, https://orcid.org/0000-0002-5238-4494 Adquisición, análisis e interpretación de los datos, revisión y edición del manuscrito final. Aprobación final de la versión a ser publicada.

Álvaro Niggemeyer, https://orcid.org/0000-0003-09116029 Adquisición, análisis e interpretación de los datos, revisión y edición del manuscrito final. Aprobación final de la versión a ser publicada.

Este artículo fue aceptado para su publicación por: Editor jefe Dr. Gerardo Soca.

\section{Bibliografía}

1. Wu Y, Ho W, Huang Y, Jin DY, Li S, Liu SL, et al. SARS-CoV-2 is an appropriate name for the new coronavirus. Lancet 2020; 395(10228): 949-50. doi: $10.1016 / \mathrm{S} 0140-6736(20) 30557-2$

2. World Health Organization. Coronavirus (COVID19) events as they happen. Geneva: WHO;2020 [consulta 23 May 2020]. Disponible en: https://www. who.int/emergencies/diseases/novel-coronavirus2019

3. Marijon E, Karam N, Jost D, Perrot D, Frattini B, Derkenne C, et al. Out-of-hospital cardiac arrest during the COVID-19 pandemic in Paris, France: a population-based, observational study. Lancet Public Health 2020. doi: 10.1016/S2468- 2667(20)30117-1

4. De Rosa S, Spaccatorella C, Basso C, Calabró MP, Curcio A, Perrone Filardi P, et al. Reduction of hospitalizations for myocardial infarction in Italy in COVID-19 Era. Eur Hert J. 2020;41(22): 2083-8. doi: 10.1093/eurheartj/ehaa409

5. Baldi E, Sechi GM, Mare C, Canevari F, Brancaglione A, Primi R, et al. Out-of-Hospital Cardiac Arrest During the Covid-19 Outbreak in Italy. N Engl J Med. 2020. NEJMc2010418. doi: 10.1056/ NEJMc2010418

6. Uruguay bajó su mortalidad en pandemia: hubo 530 muertes menos que en 2019 [Internet]. Montevideo: El Observador; 2020 [consulta 11 Jun 2020]. Disponible en: https://www.elobservador.com.uy/nota/ uruguaybajo-su-mortalidad-en-pandemia- hubo-1-574-muertes-menos- que-en-2019-20206821280. 